\title{
Influence of the Anode and Wehnelt Voltage on the Crossover Position in the Electron Beam Welding
}

\author{
Manahil Tongov \\ Faculty of Industrial Technology \\ Technical University of Sofia \\ Sofia, Bulgaria \\ tongov@tu-sofia.bg
}

\begin{abstract}
Through the numerical modelling methods, the influence of the wehnelt voltage anode voltage on the crossover position of the electron beam welding (EBW) was monitored and analysed. The finite element method (FEM) is used to obtain the simulation results. The formation of the electron beam in the electrostatic part of an electron optic system (EOS) is described by solving the electrostatic task. To obtain the current emitted from cathode, electrical and thermal tasks are solved in the cathode node and Richardson-Dushman equation is used with Schottky correction. By using the results of the electrostatic field, the active emission spot is determined. By simulating the movement of the emitted electrons under the action of the electrostatic field, the shape of the beam is determined and the distance between the cathode and the electrostatic focus is found. The results of the influence of the wehnelt and anode voltage on the magnitude of the emission current and the position of the crossover, as well as the beam dimensions in crossover and anode outlet section at constant cathode heating current are presented.
\end{abstract}

Keywords-EBW, modelling, crossover, FEM.

\section{INTRODUCTION}

Welding technological processes are based on the introduction and thermodynamically irreversible transformation of one kind of energy into another [1], [2]. In the electron beam welding (EBW), the kinetic energy of the accelerated electrons is transformed into thermal. The main advantage of the EBW to electric arc methods is the high concentration of energy flux and respectively the high density of the heat flux in the area of interaction between the focused electron beam and weld joint. This causes several times the small energy that is introduced into the welding area, and hence a significantly lower heat influence in the heat affected zone and the weld. A keyhole shape of the penetration is obtained and allows the welding of large thicknesses while at the same time achieving high welding speeds. The ratio between the width $b$ and the depth $h$ of the weld profile reaches 1:30 and more. The process of electron beam welding with the keyhole formation in the welding pool is possible at densities of the heat flow above a certain critical value [3], [4]. The electron beam area in which this density exceeds the critical is defined in [4] as the active zone, and in [5] it is shown that the quality of the weld is determined by the shape and position of the active zone relative to the welded sample. To achieve a high concentration of energy flow, the quality of the inputted to the electromagnetic focusing system electron beam is of particular importance. This beam quality is achieved in the electrostatic part of the welding apparatus. That is why the study and analysis of the processes in this part of the electron beam gun (EBG) is highly important. In this study the processes in the electrostatic part of the electron beam gun were examined and the position of the crossover was determined. The simulation modelling methods [9] and [11] provide a good opportunity to analyse the influence of the technological parameters on the physical processes. The modelling of beam-forming processes creates conditions for modelling the heat source [14]. In turn, heat sources are the basis for the modelling of the heating processes of the welded structure [13].

\section{FEM MODELLING}

To provide numerical experiments an electrostatic part of EBG, illustrated schematically in Fig. 1, is used [6] : [8] and [10]. The cathode is a strip of thickness of 0.1 [mm] and an active surface size of $2 \times 2$ [mm].

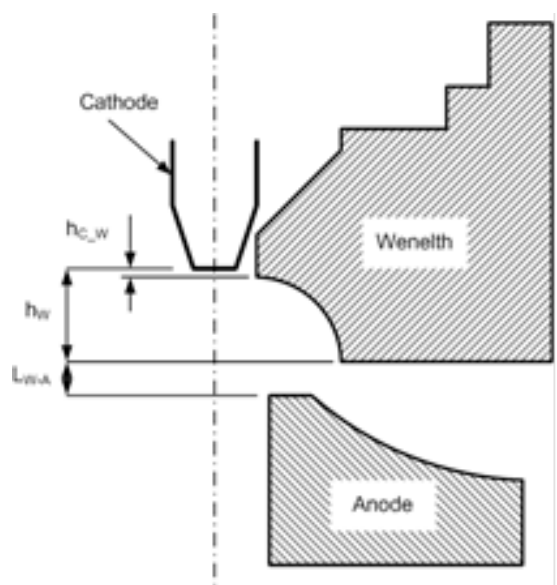

a) 


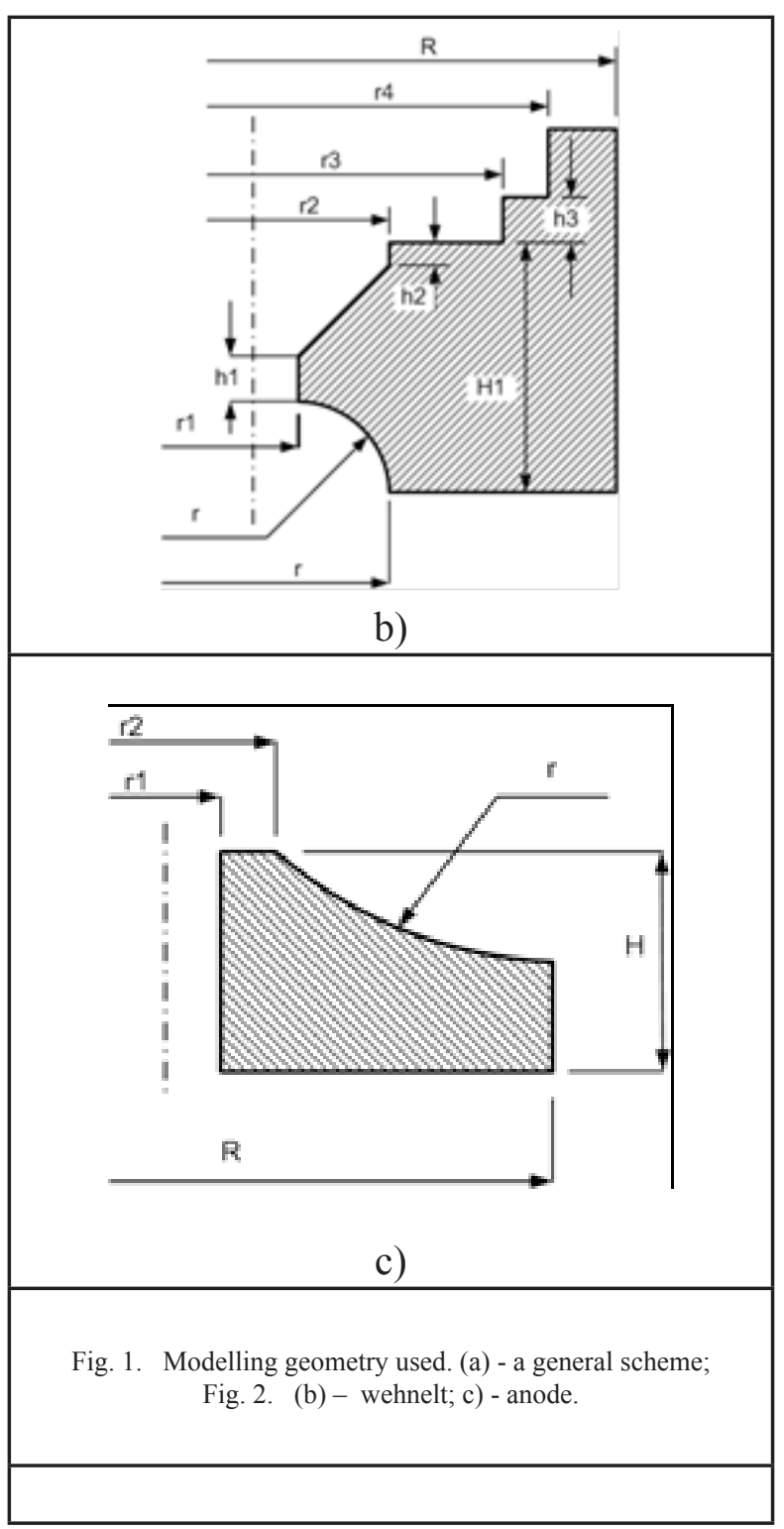

Electrons are emitted from the cathode surface. The Richardson equation describes the thermionic emission process. According to this equation, the current density of the emission is defined as:

$$
j_{R}=\beta A T^{2} \exp \left(-\frac{\varphi}{k T}\right)
$$

Here $\beta$ is a material-dependent coefficient (for tungsten the value is $0.38 \div 0.40$ [10], [12]); $\boldsymbol{T}$ - the absolute temperature; $\boldsymbol{k}$ - Boltzmann constant; $\varphi$ - work function (for tungsten $\varphi=4.3[\mathrm{eV}]$ ) and $\boldsymbol{A}$ - quantum coefficient, defined as $A=\left(4 \pi m_{e} k^{2} e\right) / h^{3}$. Here $\boldsymbol{m}_{e}$ and $\boldsymbol{e}$ are the electron mass and charge, and $\boldsymbol{h}$ is Planck's constant. Under the EBW conditions, the electrostatic field intensity $\boldsymbol{E}$ in front of the cathode has a significant effect on the emission of electrons, which makes it necessary to include the Schottky effect by using the dependence ( $\boldsymbol{E}_{n}$ is the normal to the cathode surface component of the electric field intensity):

$$
\Delta \varphi=\sqrt{\frac{e^{3} E_{n}}{4 \pi \varepsilon_{0}}}
$$

and (1) may be written as:

$$
j_{R-S}=\beta A T^{2} \exp \left(-\frac{\varphi}{k T}\right) \cdot \exp \left(\frac{1}{k T} \sqrt{\frac{e^{3} E n}{4 \pi \varepsilon_{0}}}\right)
$$

It is also noted that the emission of electrons is only performed by the active spot located on the active surface of the cathode. The condition of this is that the normal component of the electric field is directed to the cathode (Fig.2).

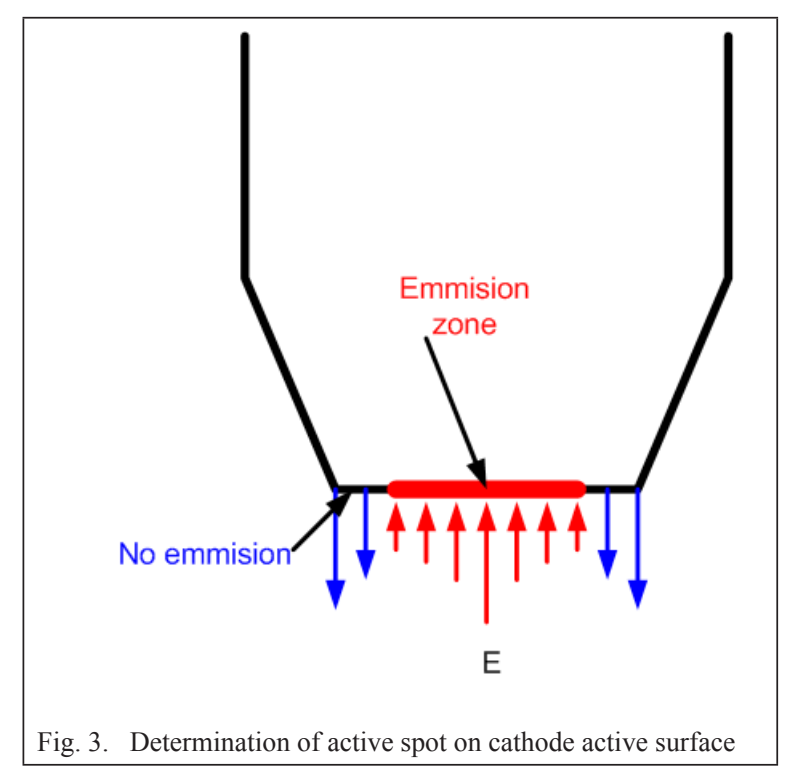

It is also possible to emit from other parts of the cathode, but these electrons fall into an electrostatic field, which returns them to the cathode. Once the electrons fall into the space between the cathode and the anode they are accelerated by the electrostatic field. The velocity vector has three components - axial, radial and orbital. The radial component leads to the formation of the crossover, and the axial has the main merit of electron energy at the anode outlet. Thus, for the simulation modelling of the beam formation, it is necessary to determine the surface temperature of the cathode and the electric field in the space between the cathode, the wehnelt and the anode. In the case in question, the cathode is directly heated (by running current). To model these processes the tasks to be solved are: electrical and thermal in the cathode and the cathode node; electrostatic and motion (of electrons) in the space between the cathode and the anode. To solve the thermal task in the cathode node, the differential equation of the heat conduction is used, and for the electric field equations for the potential and the current density in the conducting medium. For the electrostatic task, the Laplace equation was used and the potential and electric field were determined. These tasks are solved as stationary for different combinations of control and anode voltages. The control voltage is negative for the cathode and its absolute value is changed from 650 to 1150 [V]. The anode voltage is positive for the cathode and is changed from 30 to 60 [KV]. Since the parameters defining these processes do not change over time, these tasks are solved as stationary and fully coupled.

To model the electron movement in the electrostatic field, a transient task is solved and the following equations are used for each electron in the electron beam:

$$
\frac{d\left(m_{e r} \mathbf{v}_{i}\right.}{d t}=\mathbf{F}_{i}
$$




$$
m_{e r}=\frac{m_{e}}{\sqrt{1-\mathbf{v}_{i} \cdot \mathbf{v}_{i} / c^{2}}}
$$

Here $\boldsymbol{m}_{e \mathrm{r}}$ is the relativistic mass of the electron, and $\boldsymbol{F}_{\boldsymbol{i}}$ is the force acts on it. This force has two components - one resulting from the interaction with the electrostatic field and the second is electron - electron interaction. This is how it gets

$$
\mathbf{F}_{i}=-e \mathbf{E}-\frac{e^{2}}{4 \pi \varepsilon_{0}} \sum_{\substack{j=l \\ j \neq i}}^{N} \frac{\boldsymbol{r}_{\boldsymbol{i}}-\boldsymbol{r}_{j}}{\left|\boldsymbol{r}_{i}-\boldsymbol{r}_{j}\right|^{3}}
$$

To solve this task, the emission of electrons from the cathode is generated. Their initial distribution in space is based on the separation of the active spot in a number of areas. For this purpose, the mesh on the active surface of the cathode is used. The number of electrons emitted from each of these areas is proportional to the emission current of the respective area.

Factors that affect beam quality in terms of physical processes are: temperature distribution on the active surface of the cathode; the shape and dimensions of the spot through which the electrons are emitted; the structure of the electrostatic field that defines the beam section in the crossover and the anode outlet; the electron velocity components (axial, radial and orbital) of the anode outlet. We only look at the influence of two of these factors on the position of the crossover, as well as the beam dimensions in crossover and anode outlet section.

\section{RESULTS AND DISSCUTION}

In our case, the heating current is 37 [A]. Thus, the magnitude of the electron beam current is determined by the wehnelt and the anode voltage. (Fig. 3). It is calculated by integrating the current on the active surface emission:

$$
I_{\text {beam }}=\int_{S_{\text {active }}} j_{R-S}\left(T, E_{n}\right) d S
$$

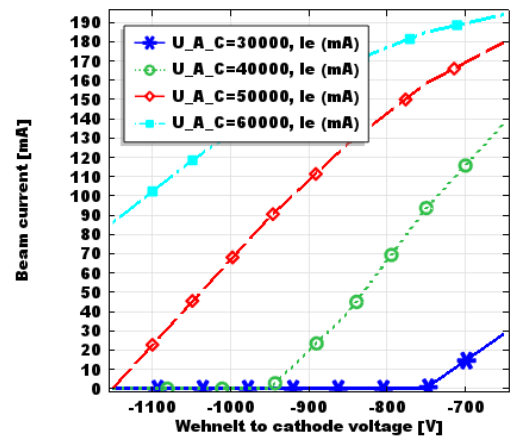

Fig. 4. . Beam current vs wehnelt voltage for different anode voltage
In calculating this integral, the current density is determined in accordance with (2). This graph shows that at low anode voltage a relatively low control voltage value is sufficient to stop the electron emission. The task of moving the electrons in the electrostatic field is solved only for the cases where a cathode emission is realized. For these cases the beam formation is monitored and the distance between the cathode and the crossover is determined. The overall shape of the beam is shown in Fig. 4. It narrows to reach the crossover and expands later. This process depends on the components of the intensity of the electric field. In Fig. 5 are given examples illustrating the influence of the anode and wehnelt voltage.

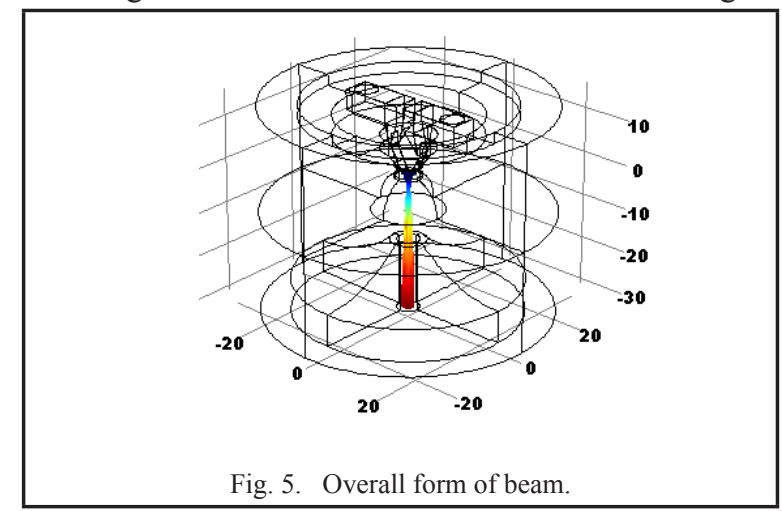

The measured values (all are in millimeters) of the distance from the cathode to the electrostatic focus are given in Table I. As the anode voltage increases, the electrostatic focus shifts to the anode. At the same time, increasing the control voltage leads to the opposite effect. The reason for this is the change in the structure of the electrostatic field with the application of these two voltages - the increase of the anode voltage leads to higher axial electron speeds and the increase of the control voltage generates higher centripetal forces. Higher axial electron velocities lead to less time to pass through the area in which the focal force acts and the focus shifts to the anode.

TABLE I. Distance From CATHODE TO CROSSOVER

\begin{tabular}{|c|c|c|c|c|c|c|}
\hline \multirow{2}{*}{$\mathbf{U}_{\mathrm{A}}$} & \multicolumn{7}{|c|}{ Wehnelt voltage $\mathbf{U}_{\mathrm{w}}[\mathbf{V}]$} \\
\cline { 2 - 7 }$[\mathbf{K V}]$ & $\mathbf{6 5 0}$ & $\mathbf{7 5 0}$ & $\mathbf{8 5 0}$ & $\mathbf{9 5 0}$ & $\mathbf{1 0 5 0}$ & $\mathbf{1 1 5 0}$ \\
\hline 30 & 0.8 & - & - & - & - & - \\
\hline 40 & 2.4 & 1.8 & 1.0 & - & - & - \\
\hline 50 & 4.2 & 3.1 & 2.3 & 1.6 & 1.0 & - \\
\hline 60 & 5.7 & 5.0 & 4.2 & 2.9 & 2.2 & 1.7 \\
\hline
\end{tabular}



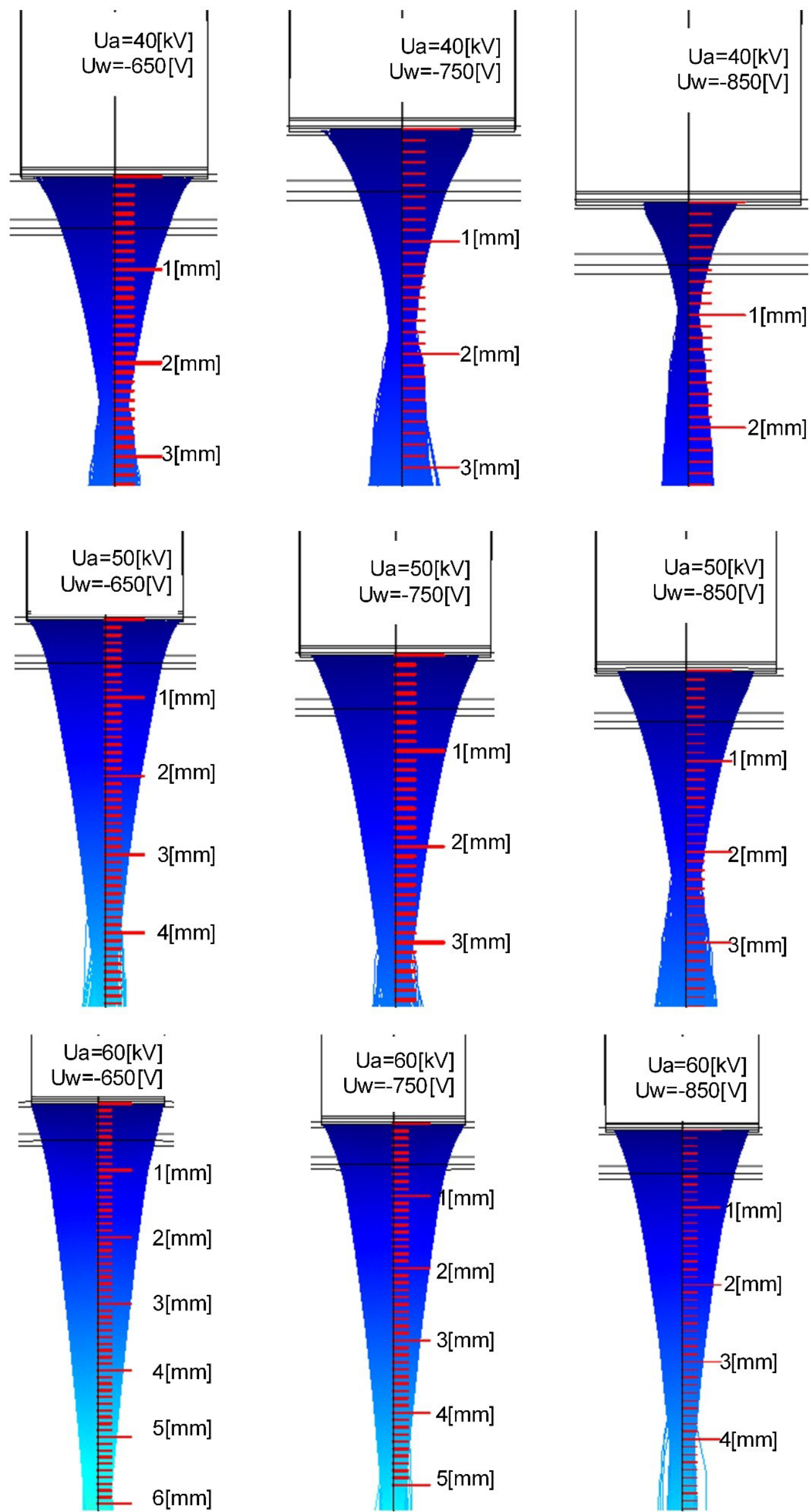

Fig. 5. Influence of anode (Ua) and wehnelt (Uw) voltage on the electron beam. 
The beam shape is examined in two sections - in the section of the crossover (blue) and the anode outlet (red) (Fig. 6). In most of the cases examined, the shape of the beam in crossover section is elliptical or oval. With the change in the parameters studied, this shape changes. Similar is the situation with regard to the shape of the beam of the anode outlet. The measured values of the beam dimensions in the indicated sections are shown in Table II and Table III. The deviation from central symmetry is mainly due to the absence of one for the cathode's active surface. These data show that with the increasing of the absolute value of the control voltage, the beam dimensions in the section decrease. The acceleration voltage influences over these dimensions in similar way. At the same time, the magnitude of the current is also decreasing (Fig. 1). To link these values, we determine the current density in the relevant sections. The magnitude of the current $\boldsymbol{I}_{b}[\mathrm{~mA}]$ represented in Fig. 3 is given in Table $I V$ for the different combinations of accelerating and control voltage. The effective radius of the beam in the crossover $\boldsymbol{r}_{\text {Ceff }}$ and on the anode outlet $\boldsymbol{r}_{\text {Aeff }}$ is calculated as:

TABLE II. BEAM DIMENSIONS IN CROSSOVER SECTION

\begin{tabular}{|c|c|c|c|c|c|c|c|c|}
\hline \multirow{2}{*}{$\begin{array}{c}\text { UW } \\
{[V]}\end{array}$} & \multicolumn{7}{|c|}{ Anode voltage, [KV] } \\
\cline { 2 - 9 } & \multicolumn{2}{|c|}{30} & \multicolumn{2}{|c|}{40} & \multicolumn{2}{|c|}{50} & \multicolumn{2}{c|}{$\boldsymbol{6 0}$} \\
\cline { 2 - 10 } & $\boldsymbol{a}$ & $\boldsymbol{b}$ & $\boldsymbol{a}$ & $\boldsymbol{b}$ & $\boldsymbol{a}$ & $\boldsymbol{b}$ & $\boldsymbol{a}$ & $\boldsymbol{b}$ \\
\hline 650 & 0.6 & 0.55 & 0.82 & 0.7 & 0.86 & 0.85 & 1.0 & 0.8 \\
\hline 750 & - & - & 0.76 & 0.62 & 0.86 & 0.79 & 0.85 & 0.85 \\
\hline 850 & - & - & 0.63 & 0.6 & 0,8 & 0.7 & 0.6 & 0.9 \\
\hline 950 & - & - & - & - & 0.74 & 0.6 & 0.88 & 0.78 \\
\hline 1050 & - & - & - & - & 0.7 & 0.58 & 0.8 & 0.7 \\
\hline 1150 & - & - & - & - & - & - & 0.7 & 0.63 \\
\hline
\end{tabular}

TABLE III. BEAM DIMENSIONS IN ANODE OUTLET SECTION

\begin{tabular}{|c|c|c|c|c|c|c|c|c|}
\hline \multirow{2}{*}{$\begin{array}{c}\text { Uw } \\
{[\mathbf{V}]}\end{array}$} & \multicolumn{9}{c|}{ Anode voltage, [KV] } \\
\cline { 2 - 9 } & \multicolumn{2}{|c|}{$\mathbf{3 0}$} & \multicolumn{2}{|c|}{$\mathbf{4 0}$} & \multicolumn{2}{|c|}{$\mathbf{5 0}$} & \multicolumn{2}{c|}{$\mathbf{6 0}$} \\
\cline { 2 - 9 } & $\boldsymbol{c}$ & $\boldsymbol{d}$ & $\boldsymbol{c}$ & $\boldsymbol{d}$ & $\boldsymbol{c}$ & $\boldsymbol{d}$ & $\boldsymbol{c}$ & $\boldsymbol{d}$ \\
\hline 650 & 0.95 & 0.8 & 2.55 & 2.35 & 2.8 & 2.8 & 2.8 & 2.8 \\
\hline 750 & - & - & 2.0 & 1.8 & 2.8 & 2.6 & 2.8 & 2.8 \\
\hline 850 & - & - & 1.1 & 0.9 & 2.4 & 2.2 & 2.85 & 2.65 \\
\hline 950 & - & - & - & - & 1.9 & 1.7 & 1.6 & 2.4 \\
\hline 1050 & - & - & - & - & 1.2 & 1.02 & 2.3 & 2.1 \\
\hline 1150 & - & - & - & - & - & - & 1.84 & 1.64 \\
\hline
\end{tabular}

$$
\begin{aligned}
& r_{\text {Ceff }}=\sqrt{a \cdot b} \\
& r_{\text {Aeff }}=\sqrt{c . d}
\end{aligned}
$$

TABLE IV

\begin{tabular}{|c|c|c|c|c|c|c|}
\hline \multirow{2}{*}{$\underset{[\mathbf{K V}]}{\mathbf{U}_{\mathrm{A}}}$} & \multicolumn{6}{|c|}{ Wehnelt voltage $U_{w}[V]$} \\
\hline & 650 & 750 & 850 & 950 & 1050 & 1150 \\
\hline 30 & 28.4 & 0 & 0 & 0 & 0 & 0 \\
\hline 40 & 137.8 & 93.7 & 39.0 & 0 & 0 & 0 \\
\hline 50 & 180.1 & 158.2 & 127.0 & 88.8 & 44.8 & 0 \\
\hline 60 & 194.3 & 184.8 & 168.7 & 146.0 & 117.9 & 86.1 \\
\hline
\end{tabular}

BEAM CURRENT
The calculated values for the current density $\boldsymbol{j}[\mathrm{mA} /$ $\left.\mathrm{mm}^{2}\right]$ in the sections considered are given in Table $V$ and Table VI. Current density in crossover increases with anode voltage increasing. When the absolute value of the control voltage increases, this density decreases, due to the dominant decrease in beam current. At the current density of the anode outlet the influence of the two voltages is more complicated. The reasons for this are the change in the distance between the two sections, the twisting of the electron beam and the deformation of the section. This issue will be discussed in a separate publication.

TABLE V. CURRENT DENSITY IN CROSSOVER SECTION

\begin{tabular}{|c|c|c|c|c|c|c|}
\hline \multirow{2}{*}{$\mathbf{U}_{\mathrm{A}}$} & \multicolumn{6}{|c|}{ Wehnelt voltage $\mathbf{U}_{\mathrm{w}}[\mathbf{V}]$} \\
\cline { 2 - 7 }$[\mathbf{K V}]$ & $\mathbf{6 5 0}$ & $\mathbf{7 5 0}$ & $\mathbf{8 5 0}$ & $\mathbf{9 5 0}$ & $\mathbf{1 0 5 0}$ & $\mathbf{1 1 5 0}$ \\
\hline 30 & 27.4 & 0 & 0 & 0 & 0 & 0 \\
\hline 40 & 76.4 & 63.3 & 32.8 & 0 & 0 & 0 \\
\hline 50 & 78.4 & 74.1 & 72.2 & 63.7 & 35.1 & 0 \\
\hline 60 & 77.3 & 81.4 & 99.4 & 67.7 & 67.0 & 62.1 \\
\hline
\end{tabular}

TABLE VI. CURRENT DENSITY IN ANODE OUTLET SECTION

\begin{tabular}{|c|c|c|c|c|c|c|}
\hline \multirow{2}{*}{$\mathbf{U}_{\mathrm{A}}$} & \multicolumn{6}{|c|}{ Wehnelt voltage $\mathbf{U}_{\mathrm{w}}[\mathbf{V}]$} \\
\cline { 2 - 7 } & $\mathbf{6 V}]$ & $\mathbf{7 5 0}$ & $\mathbf{8 5 0}$ & $\mathbf{9 5 0}$ & $\mathbf{1 0 5 0}$ & $\mathbf{1 1 5 0}$ \\
\hline 30 & 11.9 & & & & & \\
\hline 40 & 7.3 & 8.3 & 12.5 & & & \\
\hline 50 & 7.3 & 6.9 & 7.7 & 8.8 & 11.6 & \\
\hline 60 & 7.9 & 7.5 & 7.1 & 7.4 & 7.8 & 9.1 \\
\hline
\end{tabular}

\section{CONCLUTIONS}

The conclusions reached relate only to the case under consideration and cannot be said to be of a universal nature because of geometrically attached to a particular EBW installation. The main conclusions that can be drawn are the following.

1. The results of simulation modelling of the processes in the electrostatic part of EBG are presented. The influence of the anode and wehnelt voltages on the position of the crossover, the beam dimensions in crossover and anode outlet sections is determined.

1. By simulation modelling of the processes in the electrostatic part of the EBG it was found that with increasing the anode voltage the crossover moves to the anode while the increase of the control voltage leads to the opposite result.

2. In the cases considered, the distance between the cathode and the crossover varies from 0.8 to 5.7 $[\mathrm{mm}]$ depending on the control and anode voltages.

3. The current density in the crossover increases with increasing the anode voltage. When the absolute value of the control voltage increases, this density decreases, due to the dominant decrease in beam current.

4. Density of beam current in the crossover in the combinations of control and anode voltages ranged from 27 to $77\left[\mathrm{~mA} / \mathrm{mm}^{2}\right]$.

5. The beam size of the anode outlet changes from 0.8 to $2.8[\mathrm{~mm}]$ depending on the value of the variable parameters. 


\section{REFERENCES}

[1] S. David, T. Debroy, "Current Issues and Problems in Welding Science", Science Vol.257 Issue 5069, pp. 497-502, (1992), DOI: 10.1126/science. 257.5069 .497

[2] S. Kou "Welding Metallurgy" A John Wiley\&Sons, Inc.Publication, (2002), 460p., ISBN 978-0-471-28496-3

[3] G. Mladenov "Electron and ion technologies", Prof. Marin Drinov Publishing House of Bulgarian Academy of Scienses, 2009, 387p., ISBN 978-954-322-345-9.

[4] Y.Arata "Plasma, Electron and Laser Beam Technology" American Society for Metals, (1986) 630p., ISBN-13: 978-0871702548

[5] Y. Arata, K. Terai and S. Matsuda, Transaction of JWRI, Vol. 2, n.1(1973)103.

[6] S. Shiller U. Heisig and S. Panzer "Elektronenstrahltechnologie" Forschungsinstitut Manfred von Ardene, Dresden, VEB Verlag Technik, (1972)528p

[7] V. Adam et al.: "Elektronenstrahlschweißen". pro-beam AG \& Co. KgaA, München (2011).

[8] P. Petrov and S. Sabchevski "Parameters used for electron beam welding - A comparative study" Proc. 8th Int. Conf. Beam Technology, Halle, pp. 92-94, (2010).

[9] M. Dulău and L. David, "Modeling and simulation of electron's trajectory inside of electron beam gun", CEAI, Vol. 9, No. 1, pp. 27-32, 2007.
[10] M. Tongov and P. Petrov, "Study of physical processes in optical system for electron beam welding", TEMEL-ij, Valume 1, issue 2, oct. 2017. pp 85-94, ISSN 2545 - 4390

[11] V. Shcherbakov, M. V. Ivashchenko, A. S. Kozhechenko, and M. S. Gribkov, "Parametric Analysis in the Design of Technological Electron Beam Guns", ISSN 1068-3712, Russian Electrical Engineering, 2016, Vol. 87, No. 1, pp. 41-45. (C) Allerton Press, Inc., 2016, doi.org/ 10.3103/ S1068371216010089.

[12] E. Lassner and W. Schubert, "Tungsten Properties, Chemistry, Technology of the Element, Alloys, and Chemical Compaunds", ISBN 978-1-4613-7225-7, Springer Science+Business Media New York, 1999

[13] P. Lacki, K. Adamus, K. Wojsyk, M. Zawadzki and Z. Nitkiewicz, "Modeling of heat source based on parameters of electron beam welding process", ARCHIVES OF METALLURGY AND MATERIALS Volume: 56 Issue: 2 Pages: 455-462 DOI: 10.2478/ v10172-011-0049-1

[14] Y. Seregin, A. Murygin, V. Laptenok and V. Tynchenko, "Modeling of electron beam distribution in electron beam welding", IOP Conference Series: Materials Science and Engineering, Volume 450, Issue 3, 30 November 2018, Article number 03203, doi:10.1088/1757-899X/450/3/032036

\section{ACKNOWLEDGEMENTS:}

This work was supported by the Bulgarian National Scientific Fund under Grant DN 07/26 